\title{
Relative contributions of advanced glycation and nitric oxide synthase inhibition to aminoguanidine-mediated renoprotection in diabetic rats
}

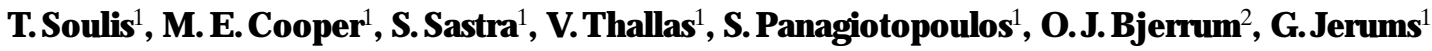 \\ ${ }^{1}$ Department of Medicine and Endocrine Unit, University of Melbourne, Austin and Repatriation Medical Centre (Repatriation \\ Campus), West Heidelberg, Australia \\ ${ }^{2}$ Pharmaceutical Biotechnology, Novo-Nordisk A/S, Bagsvaerd, Denmark
}

Summary Advanced glycation end products (AGEs) have previously been shown to be increased in the diabetic kidney. Aminoguanidine, an inhibitor of advanced glycation, has been shown to attenuate the development of AGEs as well as the progression of renal disease in experimental diabetes. However, the precise mechanisms through which aminoguanidine acts remain to be elucidated since it is also able to act as an inhibitor of nitric oxide synthase (NOS). This study has therefore compared the effects of aminoguanidine with the effects of two other inhibitors of NOS, L-NAME and methylguanidine, on the development of experimental diabetic nephropathy. Diabetic rats were randomised to receive no treatment, aminoguanidine $(1 \mathrm{~g} / \mathrm{l}$ in drinking water), L-NAME $(5 \mathrm{mg} / \mathrm{l}$ in drinking water) or methylguanidine $(1 \mathrm{~g} / \mathrm{l}$ in drinking water). Diabetic rats had increased levels of albuminuria and urinary nitrite/nitrate excretion when compared to control rats. Renal AGEs measured by fluorescence as well as by a carboxymethyllysine reactive radioimmunoassay, were elevated in diabetic rats. No changes in inducible NOS (iNOS) protein expression were detected in experimental diabetes nor did aminoguanidine affect
iNOS expression. Aminoguanidine did not affect blood glucose or $\mathrm{HbA}_{1 \mathrm{c}}$ but it did prevent increases in albuminuria, urinary nitrites/nitrates and renal AGE levels as measured by fluorescence and radioimmunoassay. L-NAME and methylguanidine did not retard the development of albuminuria, nor did they prevent increases in renal AGE levels, as assessed by fluorescence. However, these treatments did prevent increases in AGEs, as measured by radioimmunoassay. This study indicates that the renoprotective effect of aminoguanidine in experimental diabetes cannot be reproduced by L-NAME or methylguanidine. It is likely that the effect of aminoguanidine is mediated predominantly by decreased AGE formation rather than via NOS inhibition. It also raises the possibility that inhibition of fluorescent AGE formation may be more renoprotective than inhibition of the formation of carboxymethyllysine-containing AGEs. [Diabetologia (1997) 40: 1141-1151]

Keywords Advanced glycation end products, aminoguanidine, methylguanidine, L-NAME, nitric oxide, diabetic nephropathy
Received: 10 April 1997 and in revised form: 18 June 1997

Corresponding author: Dr. T. Soulis, Department of Medicine and Endocrine Unit, University of Melbourne, Austin and Repatriation Medical Centre (Repat. Campus) Banksia Street, West Heidelberg, Australia 3081

A bbreviations: AGEs, Advanced glycation end products; AG, aminoguanidine; MG, methylguanidine; L-NAME, nitro-Larginine methyl ester; NO, nitric oxide; DN, diabetic nephropathy; CML, carboxymethyllysine; GFR, glomerular filtration rate; $\mathrm{BSA}$, bovine serum albumin; $\mathrm{DAB}$, diaminobenzidine tetrahydrochloride.
The process of advanced glycation leads to irreversible cross-linking of long-lived proteins [1]. This process is accentuated in diabetes and has been postulated to contribute to the development of a range of diabetic complications including nephropathy [2], retinopathy [3] and neuropathy [4]. Aminoguanidine $(A G)$ has previously been shown to retard the development of advanced glycation end products (AGEs) in various tissues in streptozotocin diabetes [5]. In those studies, AG was shown to decrease mesangial expansion and albuminuria, both markers of the 
progression of diabetic renal disease. In man, AG is currently in Phase II/III clinical trials [6] and has already been shown to lower Hb-AGE levels [7]. However, the exact mode of action of $A G$ remains to be fully delineated. AG has high structural homology with $\mathrm{L}$-arginine, the substrate involved in the formation of nitric oxide (NO) and AG has also been recently shown to inhibit nitric oxide synthase (NOS) [8]. It has been postulated that in diabetes there is increased NOS activity in the kidney, and that NO blockade has been shown to reverse the early renal haemodynamic abnormalities of diabetes [9]. Therefore, it cannot be excluded that the renoprotective effects of AG may be via its action as an inhibitor of NOS.

The aim of this study was to determine the relative contributions of AGEs and pathways linked to NO in the development of experimental diabetic nephropathy. In addition, this study explored the renoprotective actions of $A G$ in order to determine whether AG exerts its effects via its capacity to inhibit NOS, the advanced glycation pathway or a combination of both. Inhibitors of each of the putative biochemical processes involved were used to treat diabetic rats for a period of 32 weeks. The agents used included $\mathrm{AG}$ and two relatively non-selective inhibitors of NOS, L-nitroarginine methyl ester (L-NAME), and methylguanidine (MG), neither of which have been previously reported to affect advanced glycation. End-points used to determine the effectiveness of inhibition and the contribution of each pathway to the development of experimental diabetic nephropathy included measurement of renal AGEs by their specific fluorescence, by radioimmunoassay and by immunohistochemical localisation of renal expression of AGEs. Assessment of various components of the NO pathway including NO expression in the kidney, and measurements of markers of renal disease including glomerular filtration rate (GFR) and urinary albumin excretion was also performed.

\section{Materials and methods}

This study was carried out over 32 weeks, a period previously shown to be associated with accumulation of AGEs in the kidney and evidence of renal disease as assessed by both functional and structural parameters [5]. Male Sprague Dawley rats weighing between 200-250 g were randomised into control or diabetic groups. The rats were rendered diabetic by intravenous injection of the pancreatic beta-cell toxin streptozotocin at a dose of $55 \mathrm{mg} / \mathrm{kg}$ body weight after an overnight fast. To be included in the study, rats had to have a blood glucose level $20 \mathrm{mmol} / \mathrm{l}$ or more one week after the induction of diabetes. Diabetic rats were then further randomised to receive either $A G$ hydrogen carbonate at $1 \mathrm{~g} / \mathrm{l}$ in the drinking water [5]
(Fluka Chemica, Buchs, Swizerland), the NOS inhibitor L-NAME, at a dosage of $5 \mathrm{mg} / \mathrm{l}$ in the drinking water which did not raise blood pressure [9], or MG at $1 \mathrm{~g} / \mathrm{l}$ in the drinking water [8]. The dose of LNAME [10] was based on previous studies by our group showing a reduction in urinary nitrate/nitrite excretion in diabetic rats which had been treated with L-NAME for 6 weeks [11]. Furthermore, this dose has been previously shown to prevent the effects of ACE inhibition on diabetic vessels, confirming that this dose is biologically efficacious [12]. All rats were killed at 32 weeks.

Throughout the study all rats were given free access to food (GR2 rat cubes, Clark King and Co, Melbourne, Australia). Diabetic rats received 2 IU ultralente insulin (Ultratard HM, Novo Industrie, Bagsvaerd, Denmark) every second day in order to maintain body weight and to improve survival over the study period. Rats were caged in groups of three. At 8 -week intervals, rats were weighed and placed in metabolic cages (Iffa Credo, L'Abresele, France) for collection of $24 \mathrm{~h}$ urine specimens. Urinary albumin excretion was measured by radioimmunoassay [13]. The interassay co-efficient of variation was $6.5 \%$ $(\mathrm{n}=48)$, at a concentration of $180 \mathrm{ng} / \mathrm{ml}$, the detection limit of the assay being $31.2 \mathrm{ng} / \mathrm{ml}$. Blood was collected at the same time from the tail vein for measurement of plasma glucose by the glucose oxidase technique [14] and for measurement of glycated haemoglobin by a high performance liquid chromatography assay (Biorad, Richmond, Calif., USA). Blood pressure was measured by tail cuff plethysmography [15] every 8 weeks and GFR was measured by a single injection of ${ }^{99} \mathrm{Tc}-\mathrm{DTPA}$ at week 32 [16]. Urinary nitrates/nitrites were measured by a colorimetric assay using the Greiss reagent in all groups at week 32 $[9,17]$. Briefly, samples were incubated with nitrate reductase in the presence of NADPH to reduce all nitrate to nitrite (Boehringer Mannheim GABH, Mannheim, Germany). After enzyme incubation, the total $\mathrm{NO}_{2}$ in each sample was measured with the Greiss reagent at $540 \mathrm{~nm}$ using a plate reader.

At week 32, rats were anaesthetized with pentobarbital sodium (Nembutal, Bomac, Asquith, Australia) and the left kidney was excised. The kidneys were immediately snap frozen in liquid nitrogen and stored at $-80^{\circ} \mathrm{C}$. As previously described [5], the left kidney was finely minced and processed to isolate glomeruli and tubules. Glomeruli and tubules were separated by differential sieving and suspended in phosphate buffered saline ( $\mathrm{pH} 7.4$ ), followed by centrifugation at $15000 \mathrm{rev} / \mathrm{min}$ for $30 \mathrm{~min}$ at $4{ }^{\circ} \mathrm{C}$. Lipid extraction of the pellet was performed by addition of $5 \mathrm{ml}$ of chloroform/methanol (2:1 vol/vol) followed by gentle shaking and standing overnight at $4{ }^{\circ} \mathrm{C}$. The upper layer was removed and the pellet was washed three times with methanol and distilled water. The pellet was then resuspended in $0.5 \mathrm{~mol} / 1$ acetic acid and 
$1 \mathrm{mg} / \mathrm{ml}$ pepsin, incubated for $18 \mathrm{~h}$ at $4{ }^{\circ} \mathrm{C}$ to remove non-collagenous proteins, and washed twice with $0.1 \mathrm{~mol} / \mathrm{l}$ calcium chloride, $0.02 \mathrm{~mol} / \mathrm{l}$ Tris-HCI ( $\mathrm{pH} 7.5$ ) and $0.05 \%$ toluene. No specific fluorescence could be detected in the supernatants at this stage of the extraction procedure. Following digestion with type IV collagenase $0.1 \mathrm{mg} / \mathrm{ml}$, (Sigma, St. Louis, Mo., USA) and proteinase K $0.1 \mathrm{mg} / \mathrm{ml}$, (Sigma) for $72 \mathrm{~h}$ at $37^{\circ} \mathrm{C}$ the samples were centrifuged at $15000 \mathrm{rev} / \mathrm{min}$ for $30 \mathrm{~min}$ at $4{ }^{\circ} \mathrm{C}$. The resulting supernatant was used to measure collagen-related fluorescence with excitation/emission at $370 / 440 \mathrm{~nm}$ [18]. The fluorescence of an enzyme blank (type IV collagenase and proteinase $\mathrm{K}$ ) was subtracted from the tissue fluorescence measurements. A colorimetric technique was used for the measurement of protein content [19] of the supernatants from glomerular and tubular fractions.

A specific radioimmunoassay was developed in our laboratory for the measurement of AGE [13]. The antibody used in this assay was directed against advanced glycated bovine serum albumin (BSA) and AGE-RNase was used for preparation of tracer and standards. AGE-RNase was iodinated using lactoperoxidase [20] and the AGE-RNase standards, generated as a result of 6 months incubation of RNase with $0.5 \mathrm{~mol} / \mathrm{l}$ glucose in $0.2 \mathrm{~mol} / 1$ phosphate buffer at $37^{\circ} \mathrm{C}$. Collagenase extracted renal homogenates, prepared as described above, were incubated with the AGE-BSA antibody and tracer for $5 \mathrm{~h}$ at room temperature. Sheep anti-rabbit IgG was then added and incubated overnight at room temperature. Precipitation of proteins was carried out by addition of $8 \%$ polyethylene glycol. The minimum concentration of AGE-RNase detectable was $31.2 \mathrm{ng} / \mathrm{mg}$ protein and the $\mathrm{CV}$ of this assay was $12.3 \%, n=21$, at a concentration of $2000 \mathrm{ng}$ AGE-RNase/mg protein. Radioactivity was measured using a gamma counter (Canberra Packard Instruments, St. Louis, Mo. USA) and the amount of AGEs present was expressed as ng AGERNase. To determine the epitope reacting with our antibody, competition curves were generated using increasing concentrations of antigens including AGE-BSA, AGE-RNase, carboxymethyllysine (CML)-collagen, CML-BSA, pentosidine, native BSA and native RNase. CML-collagen and CMLBSA were prepared as previously described [21]. The purity of the preparations was confirmed by amino acid analysis and by agarose gel electrophoresis [22].

To complement the results obtained using our radioimmunoassay and to assess the distribution of AGEs within the kidney, immunohistochemical studies were performed on formalin-fixed, paraffin embedded sections $(4 \mu \mathrm{m})$ of kidney taken from each rat group at week 32 . Sections were rehydrated and treated with $1 \% \mathrm{H}_{2} \mathrm{O}_{2} /$ methanol followed by incubation in Protein Blocking Agent (Lipshaw-Immunon,
Pittsburgh, Pa., USA) for $20 \mathrm{~min}$ at room temperature. Sections were then incubated with the antiAGE antibody described above for $30 \mathrm{~min}$ at room temperature. Sections were then washed in phosphate buffered saline (PBS) and incubated with biotinylated goat anti-rabbit immunoglobulin (DAKO, Carpinteria, Calif., USA). This was followed by another PBS wash and incubation with peroxidase conjugated streptavidin (DAKO). Localisation of peroxidase conjugates was revealed using diaminobenzidine tetrahydrochloride (DAB) as the chromogen. As a positive control for AGE immunoreactivity, sections taken from lung of a 40-week-old rat were used. As a control for anti-AGE immunoreactivity. AGEs formed by the addition of glucose and BSA proteins and incubated for more than 60 days at $37^{\circ} \mathrm{C}$ were preincubated with AGE antiserum and used in place of the AGE antiserum alone in the normal staining procedure. Sections were also incubated in the absence of the primary antibody as a further negative control.

Since AG was the most effective agent in retarding urinary albumin excretion in diabetic rats, (see results), it was postulated that although this may be due to a decrease in AGE levels, it could also relate to AG being more selective as an iNOS inhibitor than the other two relatively non-selective NOS inhibitors, L-NAME and MG. This led to the exploration of the possibility that diabetes is associated with an increase in inducible nitric oxide synthase (iNOS) protein expression and that the level of the enzyme could be affected by AG. Inducible NOS protein within the kidney was assessed by immunohistochemistry on formalin-fixed, paraffin embedded sections $(4 \mu \mathrm{m})$ of kidney taken from each rat group at week 32. Sections were rehydrated and treated with $1 \%\left(\mathrm{H}_{2} \mathrm{O}_{2}\right.$ /methanol followed by incubation in Protein Blocking Agent (Lipshaw-Immunon, Pittsburgh, Pa., USA) for $20 \mathrm{~min}$ at room temperature. Sections were then incubated with an anti-macrophage iNOS antibody (Transduction Laboratories, Lexington, K. Y. USA) for $30 \mathrm{~min}$ at room temperature. Sections were then washed in PBS and incubated with biotinylated goat anti-rabbit immunoglobulin (Vector Laboratories, Burlingame, Calif., USA). This incubation was then followed by another PBS wash and incubation with avidin and biotin horseradish peroxidase macromolecular complex (Vector Laboratories). Localisation of peroxidase conjugates was revealed using $\mathrm{DAB}$ as the chromogen. As a positive control for iNOS immunoreactivity, sections taken from skin wounds of rats aged 8 weeks were used. Sections were also incubated in the absence of the primary antibody as a negative control.

Statistical analysis. Logarithmic transformation of urinary albumin data was performed to yield a normal distribution for this parameter. The specific 
Table 1. Body weight, blood pressure and glycaemic control

\begin{tabular}{lrllrl}
\hline & $\begin{array}{l}\mathrm{n} \\
\end{array}$ & $\begin{array}{l}\text { Body } \\
\text { weight } \\
(\mathrm{g})\end{array}$ & $\begin{array}{l}\text { Blood } \\
\text { pressure } \\
(\mathrm{mmHg})\end{array}$ & $\begin{array}{l}\text { Glucose } \\
(\mathrm{mmol} / \mathrm{l})\end{array}$ & $\begin{array}{l}\mathrm{HbA}_{1 \mathrm{c}} \\
(\%)\end{array}$ \\
\hline Control & 9 & $568 \pm 22^{\mathrm{a}}$ & $122 \pm 2^{\mathrm{a}}$ & $5.3 \pm 0.2^{\mathrm{a}}$ & $2.6 \pm 0.1^{\mathrm{a}}$ \\
Diabetic & 15 & $398 \pm 23$ & $139 \pm 2$ & $25.6 \pm 1.5$ & $4.9 \pm 0.3$ \\
Diabetic + AG & 8 & $465 \pm 40$ & $134 \pm 2$ & $25.0 \pm 1.3$ & $4.7 \pm 0.5$ \\
$\begin{array}{l}\text { Diabetic + MG } \\
\text { Diabetic + }\end{array}$ & 9 & $367 \pm 10$ & $132 \pm 2$ & $29.8 \pm 0.8$ & $5.0 \pm 0.2$ \\
L-NAME & 11 & $368 \pm 23$ & $139 \pm 2$ & $23.3 \pm 0.9$ & $4.6 \pm 0.3$ \\
\hline
\end{tabular}

a $p<0.01$ vs all diabetic groups, data are means for weeks 8-32 except for body weight which is shown at week 32 only

contributions of diabetes, time and the various drug regimens and possible interactions were assessed by analysis of variance using the Statview SE Program (Brainpower, Calabasas, Calif., USA) on a Macintosh LC475 personal computer (Apple, Cupertino, Calif., USA). Comparisons among groups at specific time points were performed by Fisher's least significant difference method. A $p$ value of less than 0.05 was viewed as statistically significant. All data are shown as mean \pm SEM unless otherwise specified.

\section{Results}

Serial body weight and glycaemic control are shown in Table 1. Diabetic rats had significantly elevated plasma glucose and $\mathrm{HbA}_{1 \mathrm{c}}$ levels as compared to control rats. Diabetes was also associated with reduced weight gain over the study period. Treatment of diabetic rats with either AG, MG or L-NAME did not significantly affect body weight or glycaemic control during the study.

Blood pressure values as a mean of weeks 8-32 for each group, are shown in Table 1. Diabetic rats had modest increases in blood pressure throughout the study period when compared to control rats. Systolic blood pressure was not affected by treatment with AG, MG or L-NAME in the diabetic rats.

Diabetic rats had significantly elevated urinary $\mathrm{NO}_{2} / \mathrm{NO}_{3}$ levels at 32 weeks when compared to control rats. AG therapy as well as MG treatment prevented the increase in the levels of $\mathrm{NO}_{2} / \mathrm{NO}_{3}$ in diabetic rats as shown in Figure 1. L-NAME tended to decrease urinary nitrate/nitrite levels but this did not reach statistical significance $(p=0.09)$.

Throughout the study, diabetic rats had increased urinary albumin excretion as compared to control rats (control vs diabetic $F=99.7, p<0.001$ ). At 16 weeks, diabetic rats had a two to threefold increase in albuminuria as compared to control rats and this was increased to fivefold at 24 weeks and tenfold after 32 weeks of diabetes (Fig.2). Aminoguanidine therapy retarded the increase in urinary albumin

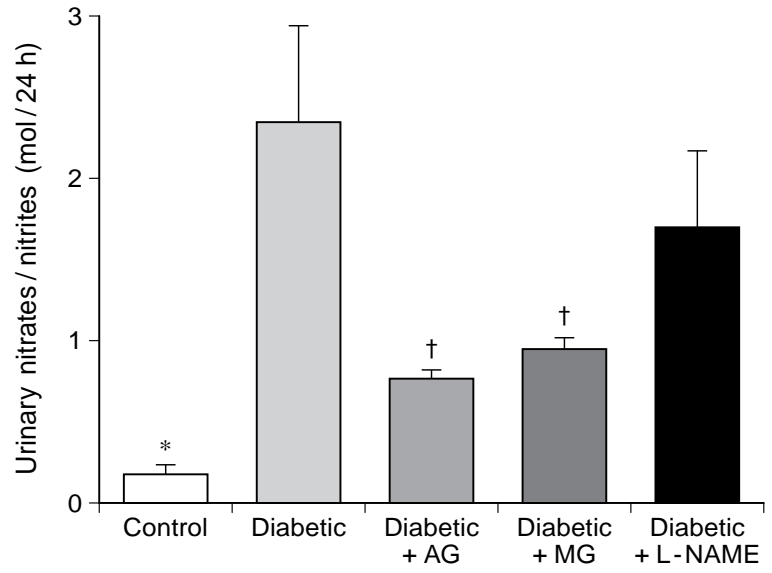

Fig. 1. Urinary levels of nitrites/nitrates are shown at 32 weeks as $\mathrm{mol} / 24 \mathrm{~h}$ for each rat group. Data are shown as mean \pm SEM. $* p<0.01$ vs all groups; $\uparrow p<0.01$ vs diabetic

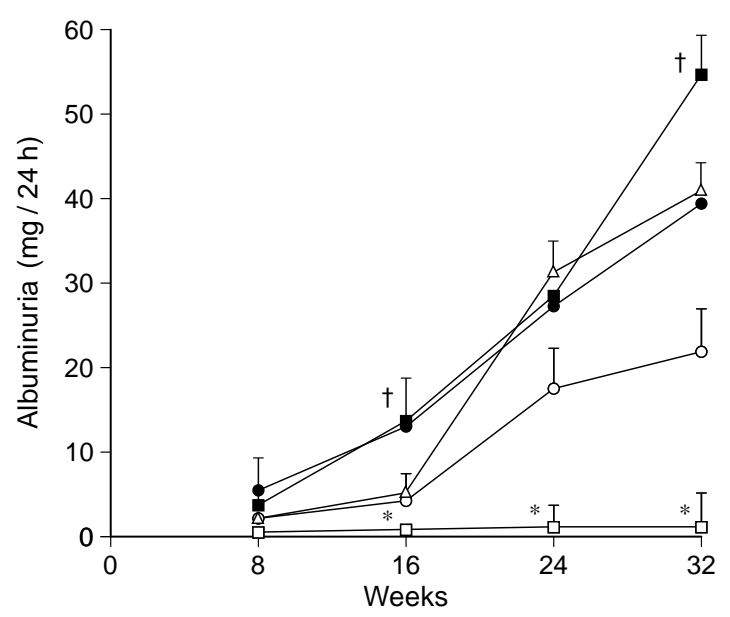

Fig. 2. Serial data for albuminuria (y-axis, logarithmic scale) are shown at 8-week intervals for control $(\square)$, diabetic ( $)$, diabetic + aminoguanidine $(\bigcirc)$, diabetic + methylguanidine $(\triangle)$ and diabetic + L-NAME $(\bullet)$. Results are shown as geometric means and tolerance factors. $* p<0.05$ vs all diabetic groups; $\uparrow p<0.05$ vs diabetic + AG

excretion at 16, 24 and 32 weeks to levels approaching but still higher than those in control rats (diabetic vs diabetic $+A G, F=8.8, p<0.01)$. Prior to 16 weeks there was no significant effect on albuminuria by $A G$ therapy. By contrast, diabetic rats treated with MG or L-NAME had sustained high levels of albuminuria over the 32 weeks (diabetic + AG vs diabetic $+\mathrm{MG}$, $\mathrm{p}=0.09 ;$ diabetic $+\mathrm{AG}$ vs diabetic $+\mathrm{L}-\mathrm{NAME}$, $F=6.7, p<0.02$ ).

At 32 weeks, diabetic rats showed an increased GFR when compared to control rats (control, $2.5 \pm 0.1 \mathrm{ml} / \mathrm{min}$ vs diabetic, $3.0 \pm 0.1 \mathrm{ml} / \mathrm{min}$, $p<0.01)$. Treatment with AG, MG or L-NAME did not significantly affect GFR (diabetic $+A G$, $3.1 \pm 0.3 \mathrm{ml} / \mathrm{min}$; diabetic $+\mathrm{MG}, 2.8 \pm 0.3 \mathrm{ml} / \mathrm{min}$; diabetic + L-NAME, $3.4 \pm 0.2 \mathrm{ml} / \mathrm{min}$, NS vs diabetic). 


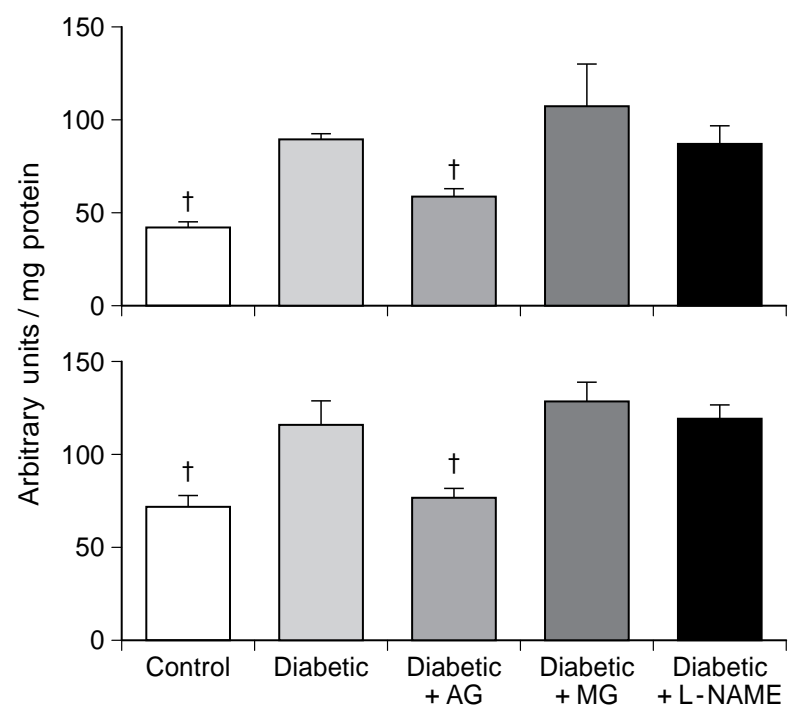

Fig. 3. Fluorescence corrected for protein content (arbitrary units/mg protein) shown for renal glomeruli (upper panel) and tubules (lower panel) for each group at 32 weeks. Results are shown as means \pm SEM. $* p<0.01$ vs diabetic

The renal content of AGEs, as measured by specific fluorescence, is shown in Figure 3. In both renal glomeruli and tubules, diabetic rats had an approximately 50 to $100 \%$ increase in fluorescent AGEs when compared to control rats. Diabetic rats, treated with AG, had levels of renal fluorescent AGEs similiar to those of control rats. Treatment of diabetic rats with either MG or L-NAME did not reduce the level of fluorescent AGEs as compared to control rats alone.

Figure 4 depicts the amount of renal AGEs for each group at 32 weeks as measured by radioimmunoassay. AGEs in both renal glomeruli and tubules were increased at least threefold and sevenfold respectively in diabetic rats as compared to control rats. Treatment of diabetic rats with $\mathrm{AG}, \mathrm{MG}$ or

Fig. 4. Levels of AGE (ng AGE-RNase/mg protein) are shown for renal glomeruli (left panel) and tubules (right panel) for each group at 32 weeks. Results are shown as means \pm SEM. $* p<0.01$ vs all groups

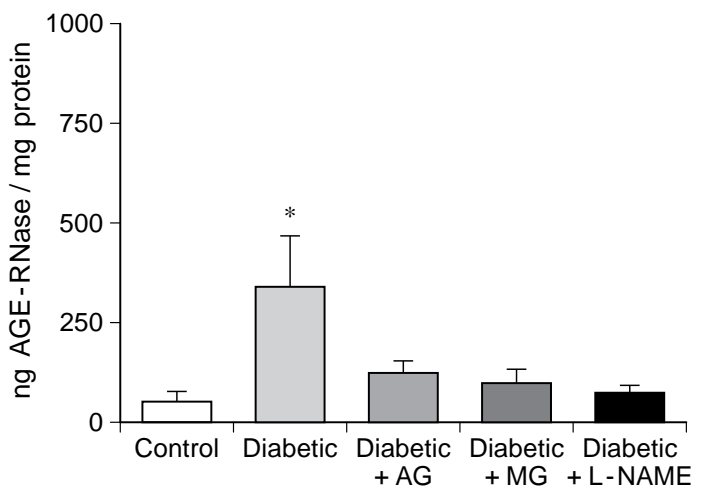

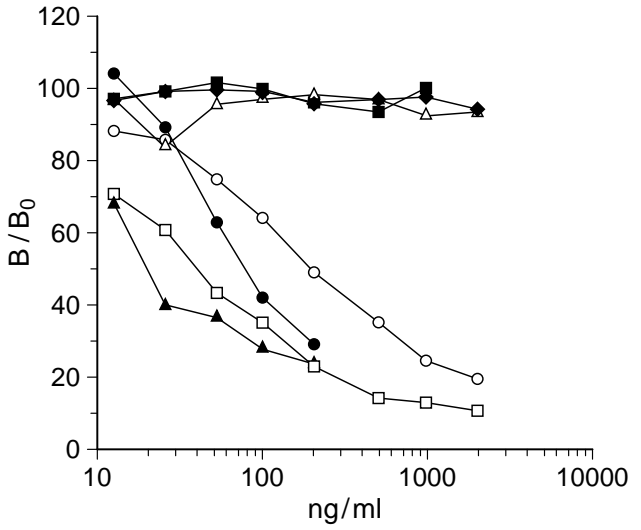

Fig. 5. Total binding curves of AGE (ng AGE-RNase/mg protein) are shown for varying concentrations of competitor antigens which include AGE-RNase $(\square)$, native BSA $(\bullet)$, AGE-BSA ( $\bigcirc)$, native RNase $(\triangle)$, pentosidine $(\square)$, CML-collagen ( $\boldsymbol{\Delta}$ ) and CML-BSA ( $\mathbf{O})$

L-NAME prevented an increase in AGE levels with values in all three groups being similar to those seen in control rats. Figure 5 depicts competition curves to the radioimmunoassay using various antigens. These studies revealed that the antibody detects advanced glycated RNase, advanced glycated BSA and CML-containing proteins. However, the antibody did not detect native BSA, native RNase or pentosidine.

Staining for AGEs in rat glomeruli from the various study groups is shown in Figure 6. There was staining within glomeruli, as well as in distal tubules and collecting ducts but there did not appear to be significant staining in proximal tubules. Diabetes was associated with increased intensity of staining, readily detected within glomeruli (Fig. 6). Treatment of diabetic rats with AG, L-NAME and MG prevented diabetes-related increases in glomerular staining for AGEs which was similar to that observed in control rats (Fig. 6A). As a positive control, staining for AGEs in the rat lung was localised to the interstitium with prominent staining of alveolar macrophages.

Immunohistochemical staining for iNOS in the kidneys from each group is shown in Figure 7. There was staining within the glomerulus for iNOS. There

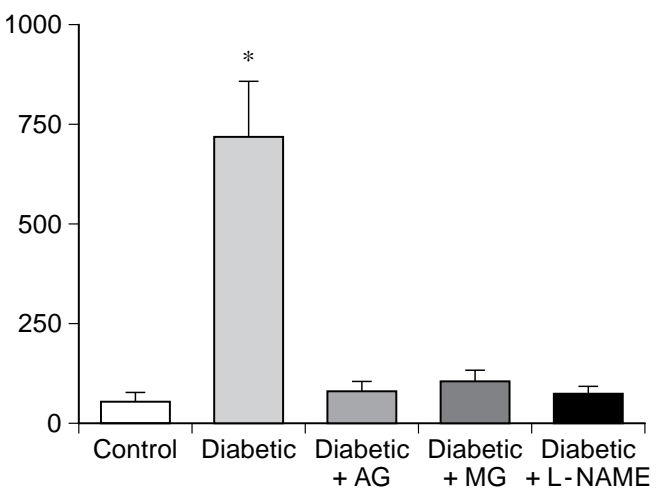



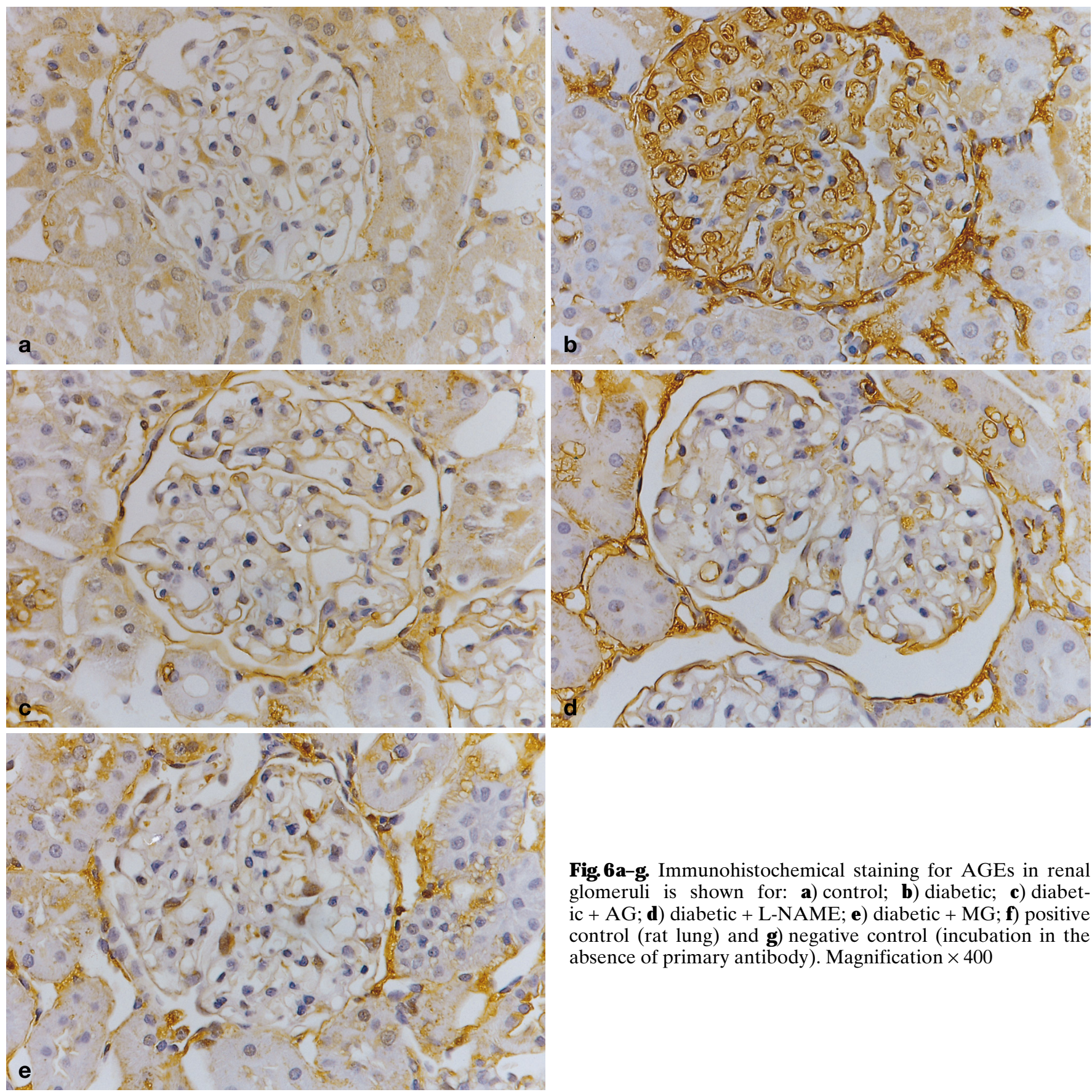

Fig.6a-g. Immunohistochemical staining for AGEs in renal glomeruli is shown for: a) control; b) diabetic; c) diabetic + AG; d) diabetic + L-NAME; $\mathbf{e}$ ) diabetic + MG; f) positive control (rat lung) and g) negative control (incubation in the absence of primary antibody). Magnification $\times 400$
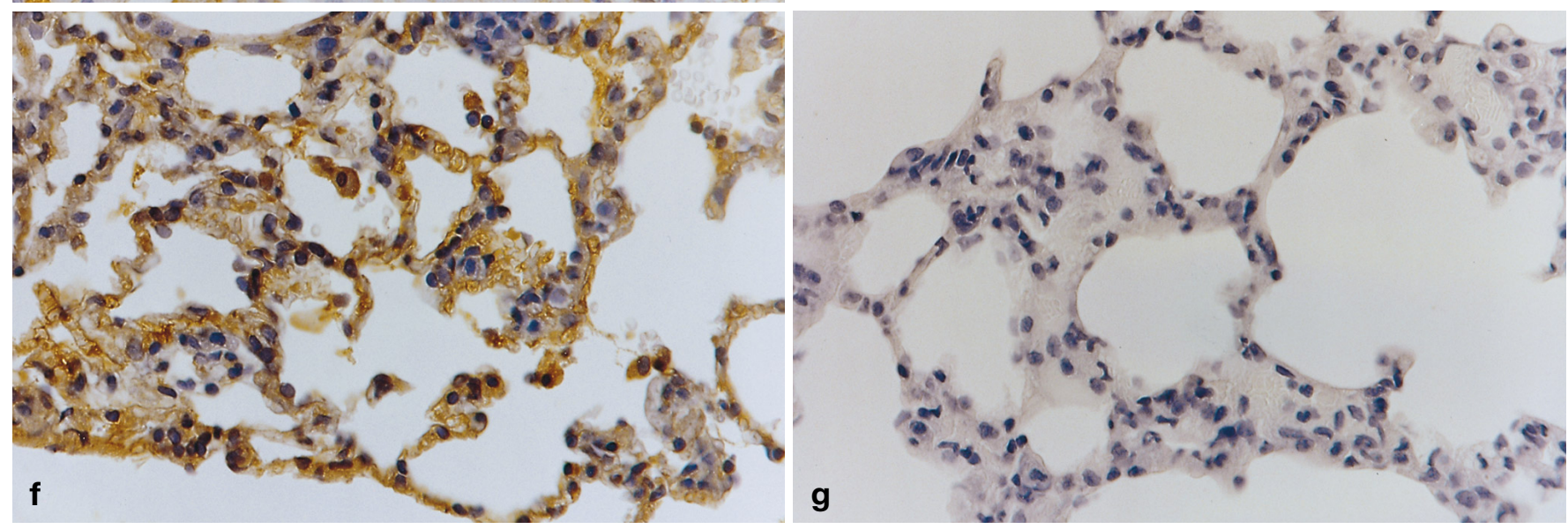

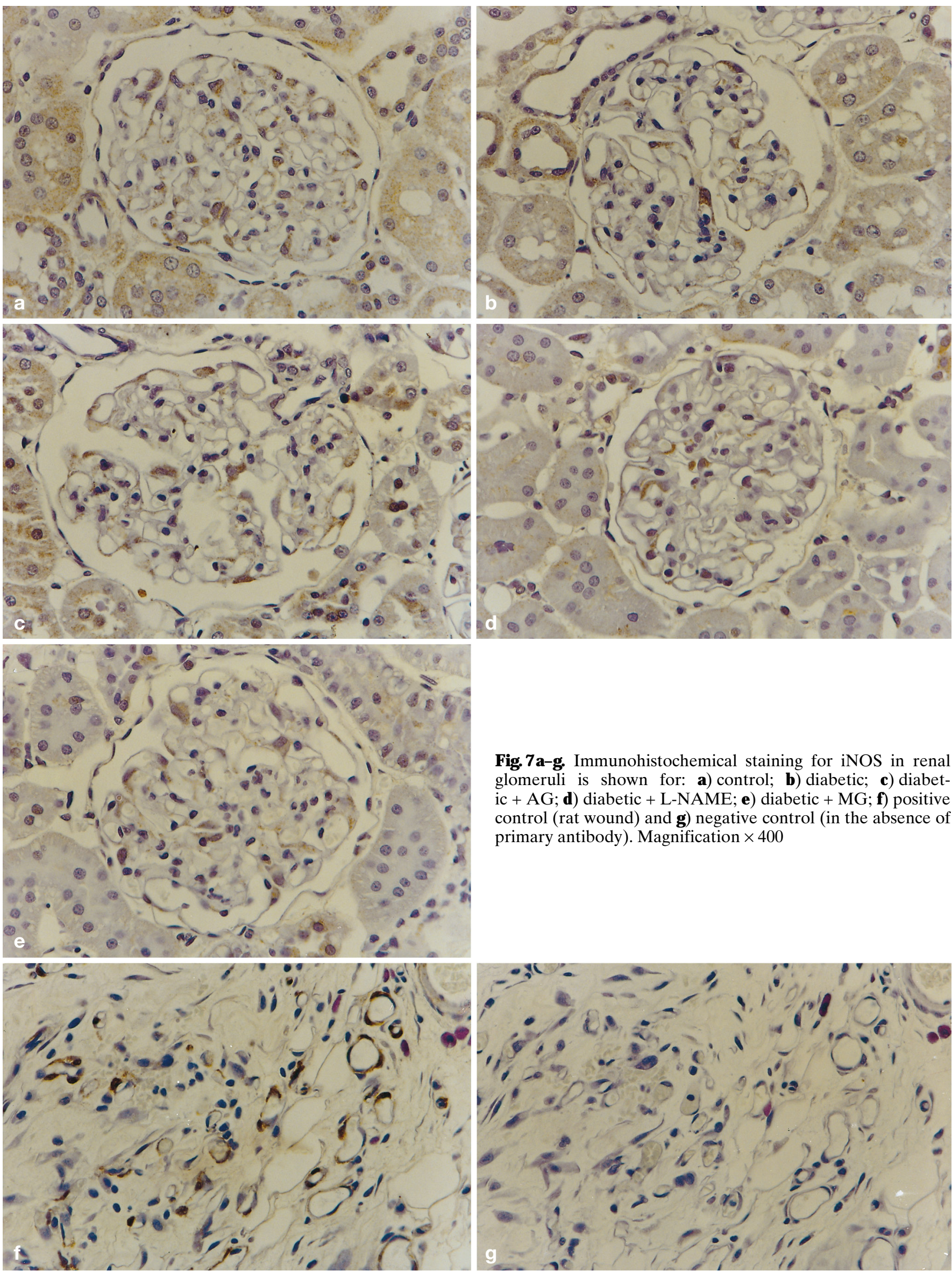

Fig.7a-g. Immunohistochemical staining for iNOS in renal glomeruli is shown for: a) control; b) diabetic; c) diabetic + AG; d) diabetic + L-NAME; e) diabetic + MG; f) positive control (rat wound) and $\mathbf{g}$ ) negative control (in the absence of primary antibody). Magnification $\times 400$

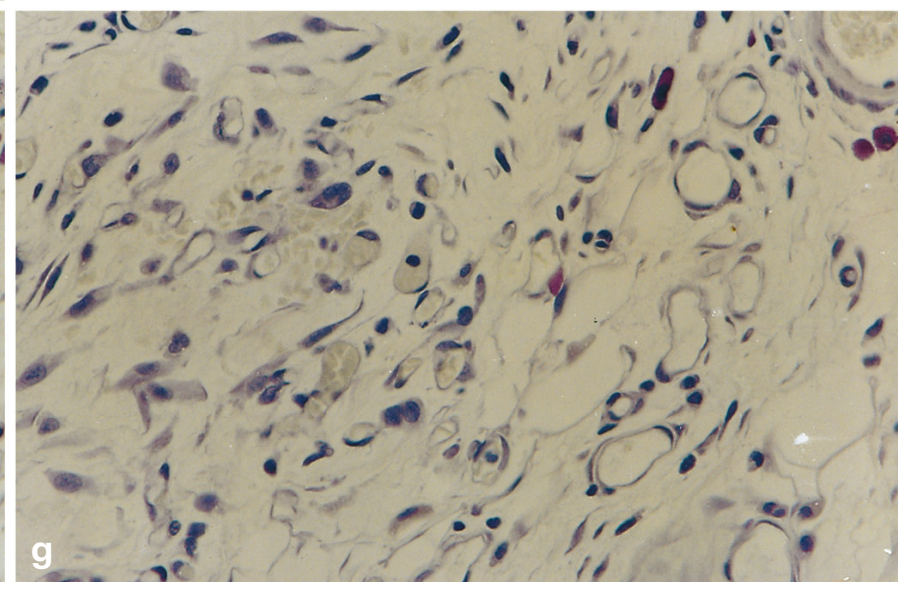


was no significant staining of renal tubules. There appeared to be no difference in the intensity or pattern of staining among the five different groups. Staining of the positive control wounds for iNOS revealed specific staining on endothelial cells.

\section{Discussion}

This study has confirmed that AG retards the progression of experimental diabetic renal disease, as assessed by albuminuria. Furthermore, AG therapy was associated with a decrease in AGEs as measured either by their specific fluorescence or by a CML-reactive radioimmunoassay. This confirms previous reports of AG effects on tissue AGE levels as measured by specific fluorescence [5] or by immunoassay [23, 24]. The process of advanced glycation has been implicated in the development of diabetic complications $[25,26]$. The resultant products known as AGEs are increased in diabetic patients [27] and have been shown to be retarded by AG treatment both in experimental diabetes [28] and in man [7]. In experimental diabetes, AG therapy has been shown to retard the development not only of albuminuria but also of mesangial expansion [5].

Although it has been previously suggested that AG acts primarily via inhibition of AGE formation, AG has also been shown to inhibit the inducible form of NOS in vitro [29] and more recently, in vivo $[30,31]$. NO, an endothelium derived relaxing factor, induces smooth muscle relaxation [32]. The status of NO in the diabetic kidney has not been clearly delineated. Our own group as well as others have shown that experimental diabetes is associated with an increase in urinary nitrite/nitrate levels $[9,10]$ and cyclic GMP levels [32] which are both markers of renal NO activity. Enhanced in vivo NO production has also been recently noted in the spontaneously diabetic BB rat, as measured by urinary excretion of nitrite [33]. These data have been interpreted as representing increased NO production within the diabetic kidney [9]. However, other factors such as food intake could also influence urinary nitrite/nitrate results [32]. In a previous study [34], diabetic rats fed a diet of L-arginine for 14 weeks had significantly decreased proteinuria, suggesting that NO supplementation rather than blockade is renoprotective. The localisation of iNOS protein within the glomerulus is consistent with previous reports of iNOS expression using other techniques including in situ hybridisation [35] and RT-PCR [36]. In the present study, renal iNOS protein expression was not altered in diabetic rats and was not affected by AG therapy. This does not exclude alterations in iNOS activity in diabetes since immunohistochemical studies detect differences in enzyme protein rather than enzyme activity.
AG has been shown in vivo to reduce NOS activity [37] and this would be consistent with the findings in the present study showing a decrease in urinary nitrite/nitrate levels. There was no effect of L-NAME on iNOS protein levels as assessed by immunohistochemistry consistent with the effect of this agent being primarily on enzyme activity. In our study, GFR was not affected by any of the interventions. In particular, a low non-pressor dose of L-NAME did not reduce GFR in the diabetic rats. This contrasts with previous studies of the effects of L-NAME on GFR in experimental diabetes [32]. However, those studies were either acute studies or were performed after only a short duration of diabetes $[38,39]$. It is possible that sensitivity to the effects of NO blockade in the kidney is reduced after a longer duration of experimental diabetes, as has been observed in other sites including the mesenteric artery and aorta [40, 41].

In our study, renal AGEs measured by fluorescence were normalised by AG treatment but not by L-NAME or MG. By contrast, AGEs measured by a specific CML-radioimmunoassay were maintained at control levels in all three intervention groups. This pattern was complemented by immunohistochemical data which clearly showed an increase in AGE staining in the kidneys from diabetic rats and prevention of renal AGE accumulation in kidneys from all three treatment groups. This suggests that NO dependent mechanisms contribute to the development of immunoreactive AGEs in the diabetic rat kidney. The discrepancy between fluorescent and radioimmunoassayable AGEs most likely relates to the measurement of different AGEs by the two methods. It is known that not all AGEs are fluorescent [42]. Products such as pentosidine and pyrraline do not fluoresce [27] and the antibody used in the radioimmunoassay in this study detects an epitope on the CML molecule (Fig.5). Furthermore, fluorescent AGEs such as pentosidine are not detected using our antiAGE antibody (Fig.5). The ability of MG and LNAME to reduce AGEs as measured by radioimmunoassay but not by fluorescence, is consistent with these agents reducing non-fluorescent AGEs such as CML. Although it had been previously reported that MG does not affect AGE levels [8], more recent data by the same group suggest that this agent reduces pentosidine levels in the aorta [43]. It is unlikely that the reduction in AGE levels by MG using the radioimmunoassay is due to effects on pentosidine since this AGE is fluorescent [25], is not detected by the radioimmunoassay used in the present study (Fig.5) and is only present at very low levels in the kidney [43].

The formation of AGE also involves oxidation and the process has been termed glucoxidation [44]. NO is known to promote oxidation and peroxynitrite production [45]. Peroxynitrite, which is a result of the reaction of $\mathrm{NO}$ and superoxide has been shown to be 
cytotoxic and to promote oxidative damage. This reaction occurs in vivo [46, 47], but the importance of this process in diabetes has not been specifically examined. Therefore, it is possible that NO dependent mechanisms involved in oxidation participate in the genesis of glycoxidation products such as CML. The concept that certain compounds inhibit glucoxidation products without influencing advanced glycation of proteins has been suggested by several investigators $[48,49]$. The results of the present study also suggest that the renoprotective effect of AG is specifically related to inhibition of the formation of fluorescent AGEs and not CML as measured by radioimmunoassay, since MG and L-NAME prevented increases in the latter without influencing albuminuria. To validate this concept further, characterisation of the chemical identity of fluorescent and non-fluorescent AGEs will be required. MG inhibits constitutive NOS but has minimal effects on AGE formation and iNOS activity [8]. In our study, MG was not as effective as AG in retarding albuminuria despite showing a similar suppression of urinary nitrate/nitrite excretion. This decreased efficacy of MG compared to AG has recently been reported in another study which evaluated these agents over 4 months in streptozotocin diabetic Sprague Dawley rats [43].

In our study, AG therapy prevented diabetes related increases in urinary nitrate/nitrite levels which were similar to levels seen in control rats, consistent with effective inhibiton of NO synthesis. Hasan et al. [50] have demonstrated that AG is a selective inhibitor of iNOS in the rat beta-cell insulinoma cell line RINm5F and also in isolated pancreatic islets. The effects of AG on blood flow and vascular albumin permeability in diabetic rats have been shown to be restricted to tissues undergoing diabetic vascular injury [51]. AG did not show effects on vascular function in the same tissues from non-diabetic rats. It was concluded that diabetes induced a selective increase in NO synthesis in tissues that are sites of complications and that AG prevented this. By contrast, other recent studies show that AGEs quench $\mathrm{NO}$ although the mechanisms by which this occurs are yet to be elucidated. For example, Bucala's group [52, 53] showed that experimental diabetes is associated with an impaired vasodilatory response to both endotheliumdependent (acetylcholine) and endothelium independent (nitroglycerine) agents, a finding which is consistent with AGE mediated inactivation of $\mathrm{NO}$ within the subendothelial space. It follows then, that if AGEs mediated the effects of diabetes on the NO system, diabetes would be associated with NO deficiency rather than an excess as postulated by Williamson et al. [54].

It has also been hypothesised that the oxygen-derived free radicals generated during formation of AGEs may interfere with $\mathrm{NO}$ action and thus attenuate its vasodilatory activity [54]. In vitro studies have shown that advanced glycation of proteins impede NO availability and bioactivity in two ways: by direct inactivation (quenching) and by impairing the carrierfunction of proteins for NO [55]. This has been supported by in vivo studies suggesting that AGEs quench $\mathrm{NO}$ and that AG treatment retards this effect [52].

In our study, diabetic rats had modest increases in blood pressure when compared to control rats but this was not reduced by any of the agents employed. The only agent to significantly reduce albuminuria was AG, yet it did not influence systemic blood pressure. This is particularly relevant since blood pressure has been shown to be a major determinant of the progression of experimental diabetic nephropathy [56] and antihypertensive treatment even in the absence of systemic hypertension has been shown to retard renal injury in diabetic rats [57]. Previous studies have implicated NO-dependent mechanisms in beta-cell injury, including injury related to streptozotocin. AG has been reported to delay the onset of diabetes in the $\mathrm{BB}$ rat [33]. A similar effect was observed with the NOS inhibitor monomethyl-L-arginine (LNMMA) [33] suggesting that this effect of $A G$ on beta-cell function is via its action as an NOS inhibitor. In the present study, there was no evidence of an effect of AG, MG or L-NAME on glycaemic control in diabetic rats.

Recent in vivo and in vitro studies have provided further evidence to link AGE formation to renal injury $[58,59]$. It has been demonstrated that exogenous AGEs in non-diabetic rats mimic the long-term effects of diabetes such as increased mesangial expansion [60] as well as stimulating various cytokines including transforming growth factor (TGF) $\beta 1$. Recent studies by our group suggest that a similar phenomenon occurs in diabetic vessels and that this increase in TGF $\beta 1$ and in gene expression of matrix type IV collagen can be inhibited by AG [23]. Thus, it appears likely that the predominant action of $\mathrm{AG}$ as a renoprotective agent is via inhibition of the process of advanced glycation rather than via inhibition of inducible NOS and may involve prevention of AGE-induced increases in cytokine production.

A cknowledgements. The authors wish to thank Leanne Thomas for technical assistance. This work was supported by grants from Juvenile Diabetes Foundation International, Hoechst Diabetes Research and Development Board, the Australian Kidney Foundation and the National and Central Health and Medical Research Councils of Australia.

\section{References}

1. Monnier V, Vishwanath V, Frank K, Elmets G, Dauchot P, Kohn R (1986) Relation between complications of type I diabetes mellitus and collagen-linked fluorescence. N Eng J Med 314: 403-408 
2. Nicholls K, Mandel T (1989) Advanced glycosylation end products in experimental murine diabetic nephropathy: effect of islet grafting and of aminoguanidine. Lab Invest 60: 486-489

3. Hammes H, Martin S, Federlin K, Geisen K, Brownlee M (1991) Aminoguanidine treatment inhibits the development of experimental diabetic retinopathy. Proc Natl Acad Sci USA 88: 11555-11558

4. Cameron NE, Cotter MA, Dines K, Love A (1992) Effects of aminoguanidine on peripheral nerve function and polyol pathway metabolites in streptozotocin-diabetic rats. Diabetologia 35: 946-950

5. Soulis-Liparota T, Cooper ME, Papazoglou D, Clarke B, Jerums G (1991) Retardation by aminoguanidine of development of albuminuria, mesangial expansion, and tissue fluorescence in streptozocin-induced diabetic rat. Diabetes 40: 1328-1334

6. Bucala R, Vlassara H (1995) Advanced glycosylation end products in diabetic renal and vascular disease. Am J Kidney Dis 26: 875-888

7. Makita Z, Vlassara H, Rayfield E, et al. (1992) Hemoglobin-AGE: a circulating marker of advanced glycosylation. Science 258: 651-653

8. Tilton RG, Chang K, Hasan KS, et al. (1993) Prevention of diabetic vascular dysfunction by guanidines. Inhibition of nitric oxide synthase versus advanced glycation end-product formation. Diabetes 42: 221-232

9. Komers R, Allen TJ, Cooper ME (1994) Role of endothelium-derived nitric oxide in the pathogenesis of the renal hemodynamic changes of experimental diabetes. Diabetes 43: $1190-1197$

10. Bank S, Aynedjian H (1993) Role of EDRF (nitric oxide) in diabetic renal hyperfiltration. Kidney Int 43: 1306-1312

11. Nguyen D, Panagiotopoulos S, Soulis T, Cooper M, Jerums $G$ (1995) Aminoguanidine and methylguanidine reduce mesenteric vascular enlargement in experimental diabetes. Diabetologia 38 [Suppl 1]: A255 (Abstract)

12. Rumble J, Komers R, Cooper M (1995) Kinins or nitric oxide, or both, are involved in the antitropic effects of angiotensin converting enzyme inhibitors on diabetes-associated mesenteric vascular hypertrophy in the rat. J Hypertens 14: 601-607

13. Soulis T, Cooper ME, Vranes D, Bucala R, Jerums G (1996) The effects of aminoguanidine in preventing experimental diabetic nephropathy are related to duration of treatment. Kidney Int 50: 627-634

14. Schmidt F (1961) Enzymatic determination of glucose and fructose simultaneously. Klin Woch 39: 1244-1247

15. Bunag R (1973) Validation in awake rats of a tail-cuff method for measuring systolic pressure. J Appl Physiol 34: 279-282

16. Allen TJ, Cooper ME, O'Brien RC, Bach LA, Jackson B, Jerums G (1990) Glomerular filtration rate in streptozocin-induced diabetic rats. Role of exchangeable sodium, vasoactive hormones, and insulin therapy. Diabetes 39: 11821190

17. Bartholomew B (1984) A rapid method for the assay of nitrate reductase enzyme of Escherichia coli. Food Chem Toxicol 22: 541-543

18. Brownlee M, Vlassara H, Kooney A, Ulrich P, Cerami A (1986) Aminoguanidine prevents diabetes-induced arterial wall protein cross-linking. Science 232: 1629-1632

19. Bradford M (1976) A rapid and sensitive method for the quantification of microgram quantities of protein utilizing the principle of protein-dye binding. Anal Biochem 72: 248-254
20. Salacinski P, McLean C, Sykes J, Clement-Jones W, Lowry P (1981) Iodination of proteins, glycoproteins and peptides using a solid phase oxidising agent, 1,3,4,6-1,3,4,6-tetrachloro-3a,6a-diphenylglycoluril. Anal Biochem 117: 136-146

21. Reddy S, Bichler J, Wells-Knecht KJ, Thorpe SR, Baynes JW (1995) N epsilon-(carboxymethyl)lysine is a dominant advanced glycation end product (AGE) antigen in tissue proteins. Biochemistry 34: 10872-10878

22. Bjerrum OJ, Ingild A, Lowenstein H, Weeke B (1973) Quantitation of human IgG by rocket immunoelectrophoresis at $\mathrm{pH} 5$ by use of carbamylated antibodies: a routine laboratory method. Clin Chim Acta 46: 337-343

23. Rumble JR, Cooper ME, Soulis T, et al. (1997) Vascular remodeling in experimental diabetes: role of advanced glycation end products. J Clin Invest 99: 1016-1027

24. Makita Z, Vlassara H, Cerami A, Bucala R (1992) Immunochemical detection of advanced glycosylation end products in vivo. J Biol Chem 267: 5133-5138

25. Sell DR, Monnier VM (1990) End-stage renal disease and diabetes catalyze the formation of a pentose-derived crosslink from aging human collagen. J Clin Invest 85: 380-384

26. Vlassara H, Bucala R, Striker L (1990) Pathogenic effects of advanced glycosylation: biochemical, biological and clinical implications for diabetes and aging. J Lab Invest 70: $138-151$

27. McCance DR, Dyer DG, Dunn JA, et al. (1993) Maillard reaction products and their relation to complications in insulin-dependent diabetes mellitus. J Clin Invest 91: 2470 2478

28. Soulis-Liparota T, Cooper ME， Dunlop M， Jerums G (1995) The relative roles of advanced glycation, oxidation and aldose reductase inhibition in the development of experimental diabetic nephropathy in the Sprague-Dawley rat. Diabetologia 38: 387-394

29. Corbett JA, Tilton RG, Chang K, et al. (1992) Aminoguanidine, a novel inhibitor of nitric oxide formation, prevents diabetic vascular dysfunction. Diabetes 41: 552-556

30. Worrall N, Misko T, Sullivan P, Hui J, Rodi C, Ferguson T (1996) Corticosteroids inhibit expression of inducible nitric oxide synthase during acute cardiac allograft rejection. Transplantation 61: 324-328

31. Mourelle M, Vilaseca J, Guarner F, Salas A, Malagelaida J (1996) Toxic dilation of colon in a rat model of colitis is linked to inducible form of nitric oxide synthase. Am J Physiol 33: G425-G430

32. Tolins JP, Shultz PJ, Raij L, Brown DM, Mauer SM (1993) Abnormal renal hemodynamic response to reduced renal perfusion pressure in diabetic rats: role of NO. Am J Physiol 265: F886-F889

33. Wu G (1995) Nitric oxide synthesis and the effect of aminoguanidine and NG-monomethyl-L-arginine on the onset of diabetes in the spontaneously diabetic BB rat. Diabetes 44: 360-364

34. Reyes A, Karl I, Kissane J, Klahr S (1993) L-arginine administration prevents glomerular hyperfiltration and decreases proteinuria in diabetic rats. J Am Soc Neph 43: 1039-1045

35. Ahn K, Mohaupt M, Madsen K, Kone B (1996) In situ hybridisation localisation of mRNA encoding iNOS in rat kidney. Am J Physiol 34: F748-F757

36. Morrisey J, McCracken R, Kaneto H, Vehaskari M, Montani D, Klahr S (1994) Location of inducible nitric oxide synthase mRNA in the normal kidney. Kidney Internat 45: 998-1005 
37. Ruetten H, Thiemermann C (1996) Prevention of the expression of inducible nitric oxide synthase by aminoguanidine or amino-ethyl-isothiourea in macrophages and in the rat. Biochem Biophys Res Comm 225: 525-530

38. King A, Zayas M, Troy J, Downes S, Brenner B (1990) Inhibition of diabetes-induced hyperfiltration and hyperemia by N-omega-monomethyl-L-arginine (L-NMMA). J Am Soc Nephrol 1: 666 (Abstract)

39. Mattar A, Fujihara C, Ribeiro M, Denucci G, Zatz R (1996) Renal effects of acute and chronic nitric oxide inhibition in experimental diabetes. Nephron 74: 136-143

40. Tesfamariam B, Brown M, Cohen R (1991) Elevated glucose impairs endothelium-dependent relaxation. J Clin Invest 87: 1643-1648

41. Meraji S, Jayakody L, Senarantine M, Thomson A, Kappagoda T (1987) Endothelium-dependent relaxation in aorta of $\mathrm{BB}$ rat. Diabetes 36: 978-981

42. Dyer DG, Dunn JA, Thorpe SR, et al. (1993) Accumulation of Maillard reaction products in skin collagen in diabetes and aging. J Clin Invest 91: 2463-2469

43. Nyengard J, Chang K, Berhorst S, Reiser K, Williamson J, Tilton R (1997) Discordant effects of guanidines on renal structure and function and on regional vascular dysfunction and collagen changes in diabetic rats. Diabetes 46: 94-106

44. Fu MX, Wells Knecht KJ, Blackledge JA, Lyons TJ, Thorpe SR, Baynes JW (1994) Glycation, glycoxidation, and cross-linking of collagen by glucose. Kinetics, mechanisms, and inhibition of late stages of the Maillard reaction. Diabetes 43: 676-683

45. Van der Vliet A, Smith D, O’Neill CA, et al. (1994) Interactions of peroxynitrite with human plasma and its constituents: oxidative damage and antioxidant depletion. Biochem J 303: 295-301

46. Haddad IY, Crow JP, Hu P, Ye Y, Beckman J, Matalon S (1994) Concurrent generation of nitric oxide and superoxide damages surfactant protein. Am J Physiol 267: L242L249

47. Rubbo H, Radi R, Trujillo M, et al. (1994) Nitric oxide regulation of superoxide and peroxynitrite dependent lipid peroxidation. Formation of novel nitrogen-containing oxidised lipid derivatives. J Biol Chem 269: 2606626075

48. Baynes JW (1991) Role of oxidative stress in development of complications in diabetes. Diabetes 40: 405-412

49. Kochakian M, Manjula B, Egan J (1996) Chronic dosing with aminoguanidine and novel advanced glycosylation end product formation inhibitors ameliorates cross-linking of tail tendon collagen in streptozotocin-induced diabetic rats. Diabetes 45: 1694-1700

50. Hasan K, Heesen B, Corbett J, et al. (1993) Inhibition of nitric oxide formation by guanidines. Eur J Pharmacol 249: 101-106

51. Corbett JA, McDaniel ML (1992) Does nitric oxide mediate autoimmune destruction of beta-cells? Possible therapeutic interventions in IDDM. Diabetes 41: 897-903

52. Bucala R, Tracey K, Cerami A (1991) Advanced glycosylation products quench nitric oxide and mediated defective endothelium-dependent vasodilation in experimental diabetes. J Clin Invest 87: 432-438

53. Hogan M, Cerami A, Bucala R (1992) Advanced glycosylation endproducts block the antiproliferative effect of nitric oxide. Role in the vascular and renal complications of diabetes mellitus. J Clin Invest 90: 1110-1115

54. Williamson JR, Chang K, Frangos M, et al. (1993) Hyperglycemic pseudohypoxia and diabetic complications. Diabetes 42: 801-813

55. Farkas J, Menzel E (1995) Proteins lose their nitric oxide stabilising function after advanced glycosylation. Biochim Biophys Acta 1245: 305-310

56. Cooper ME, Allen TJ, Macmillan P, et al. (1988) Effects of genetic hypertension on diabetic nephropathy in the rat: functional and structural characteristics. J Hypertens 6: 1009-1016

57. Zatz R, Dunn B, Meyer T, Anderson S, Rennke H, Brenner B (1986) Prevention of diabetic glomerulopathy by pharmacological amelioration of glomerular capillary hypertension. J Clin Invest 77: 1925-1930

58. Yang CW, Vlassara H, Peten EP, He CJ, Striker GE, Striker LJ (1994) Advanced glycation end products up-regulate gene expression found in diabetic glomerular disease. Proc Natl Acad Sci USA 91: 9436-9440

59. Doi T, Vlassara H, Kirstein M, Yamada Y, Striker G, Striker L (1992) Receptor specific increases in extracellular matrix productions in mouse mesangial cells by AGEP is mediated via platelet growth factor. Proc Natl Acad Sci USA 89: 2843-2877

60. Vlassara H, Fuh H, Makita Z, Krungkrai S, Cerami A, Bucala R (1992) Exogenous advanced glycation end products induce complex vascular dysfunction in normal animals. Proc Natl Acad Sci USA 89: 12043-12047 